\title{
Electro-osmotic Flow Measurement to Determine Thermodynamic Parameters in Epithelia*
}

\author{
Manabu Miyamoto, Takashi Nakahari, Hideyo Yoshida \\ and Yusuke IMAI
}

Department of Physiology, Osaka Medical College, Takatsuki, Osaka 569

(Received Nov. 15 1986)

Electro-osmotic flow and streaming potential were demonstrated in frog skin and gastric mucosa by the oscillating method (Imai, et al 1986) in a Ussing chamber ${ }^{1,2)}$. Electro-osmotic flow was monitored directly using a photodiode. The flow increased in proportion to the amplitude of the applied rectangular electropotential wave. Their ratios were $0.31 \mu 1 / \mathrm{min} / \mathrm{V} / \mathrm{cm}^{2}$ for the skin and $0.69 \mu \mathrm{l} / \mathrm{min} / \mathrm{V} / \mathrm{cm}^{2}$ for the gastric mucosa. In $\mathrm{pH} 7.4$ Ringer solution, skin behaved as a cation exchanger and gastric mucosa as an anion exchanger. The electro-osmotic flow evoked by the potential oscillation was also measured against a constant osmotic flow induced by a sucrose gradient. The electric potential which balanced the finite osmotic flow was $-241 \mathrm{mV} / 200 \mathrm{mOsm}$ for the skin and $156 \mathrm{mV} / 100 \mathrm{mOsm}$ for the gastric mucosa. The parameters of the theoretical model were also determined using the network thermodynamics. The values of the reflection coefficients $\left(\sigma_{\mathrm{Cl}}-\sigma_{\mathrm{Na}}\right)$ and hydraulic conductivities $(L p)$ were $0.14,2 \times 10^{-7} \mathrm{~cm} / \mathrm{sec} / \mathrm{atm}$ for the skin, and $-0.11,4.5 \times 10^{-7} \mathrm{~cm} / \mathrm{sec} / \mathrm{atm}$ for the gastric mucosa. The absolute values of reflection coefficients of each ion for the skin were $\sigma_{\mathrm{Cl}}=0.96$ and $\sigma_{\mathrm{Na}}=0.82$.

Key words : Electro-osmosis, Epithelium, Power coupling, Reflection coefficient, Hydraulic conductivity

\section{Introduction}

Volume flow is driven not only directly by hydrostatic pressure and osmotic pressure differences, but also by the extra driving force produced by power coupling processes. The concept of "power coupling" was introduced by Imai et al in $1981^{3,4)}$. They proposed three power domains (hydrodynamic, ionic and electric power), and treated electro-kinetic phenomena as a power coupling process between hydraulic and electric power. Streaming potential is a power transducing process from hydraulic to electric

* 上皮膜での電気浸透流測定とその熱力学的パラメータの決定

** 宮本 学, 中張隆司, 吉田秀世, 今井雄介: 大阪医科大学 第一生理学教室 ₹ 569 高規市大学町 2-7 power via ionic transport processes. Electroosmosis is a power transducing process from electric to hydraulic power via ionic transport processes (Fig. 1).

In the present paper, the power coupling of electric power with hydrodynamic power is shown directly by measuring the corresponding volume flow oscillation with the transmembrane potential oscillation. This electro-osmotic flow depends on both hydraulic conductivity $(L p)$, and the difference of reflection coefficients $(\sigma)$ between anions and cations. These parameters have characteristic values in some biological membranes. 


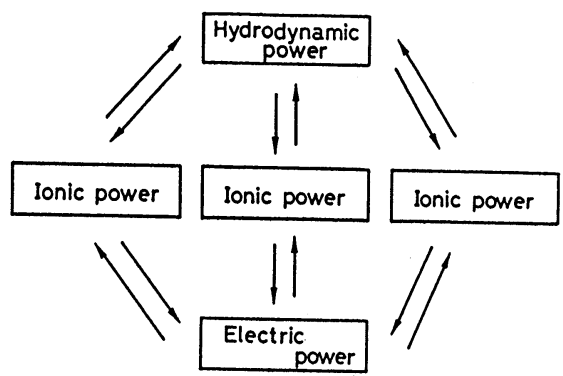

Fig. 1 Power domains in network thermodynamics (Hydrodynamic power, Ionic powers and Electric power)

"Power Couplings" are assumed to exist between hydrodynamic power and ionic powers, and between ionic powers and electric power. But there is indirect coupling between hydrodynamic power and electric power and among ionic powers.

\section{Theoretical}

The volume flow across a biological membrane has been described by Starling's hypothesis as follows.

$$
J v=L p(\Delta p-\Delta \pi)
$$

On the other hand, "coupling" between solvent and solute (nonelectrolyte) was proposed in the thermodynamic approach by Kedem \& Katchalsky ${ }^{5}$, who expressed volume flow as

$$
\begin{aligned}
& J v=L p(\Delta p-\sigma \Delta \pi) \\
& =L p\left(\Delta p-\Delta \pi+\Sigma_{s}\left(1-\sigma_{s}\right) \Delta \pi_{s}\right) \\
& =L p\left(\Delta p-\Delta \pi+\Sigma_{s}\left(1-\sigma_{s}\right) \bar{c}_{s} \Delta \mu_{s}\right)
\end{aligned}
$$

The transport equation of volume flow for electrolytes was proposed by Imai, et $\mathrm{al}^{3)}$ on the basis of network thermodynamics.

$$
J v=L p\left\{(\Delta p-\Delta \pi)+\Sigma_{i}\left(1-\sigma_{i}\right) \bar{c}_{i} \Delta \widetilde{\mu}_{i}\right\}
$$

Thus, volume flow was expressed by the product of hydraulic conductivity $(L p)$ and the sum of conjugated driving force and extra driving force caused by power coupling. Here, $\sigma_{i}$ is the reflection coefficient of $i$ ion, and $\bar{c}_{i}\left(=\Delta c_{i} / \Delta \ln \right.$ $\left.c_{i}=\Delta \pi / \Delta \mu_{i}\right)$ is the logarithmic mean concentration defined by Kedem and Katchalsky ${ }^{5}$. The electrochemical potential $\left(\Delta \widetilde{\mu_{i}}\right)$ is represented as $J v=L p\left\{(\Delta p-\Delta \pi)+\Sigma_{i}\left(1-\sigma_{i}\right) \bar{c}_{i}\left\{\Delta \mu_{i}+z_{i} F \Delta \varphi\right\}\right.$ (4) where $z_{i}$ is charge number of ion $i, F$ is Faraday constant and $\Delta \varphi$ is potential difference.

If the concentrations of solutes are equal in both chambers and there is no hydrostatic pressure difference $\left(\Delta p=0, \Delta \pi=0, \quad \bar{c}_{i}=c_{i}\right.$ and $\Delta \mu_{i}=0$ ), equation (4) can be simplified as follows.

$$
J v=L p F \Delta \varphi \Sigma_{i}\left(1-\sigma_{i}\right) z_{i} c_{i}
$$

\section{Experimental}

The experiments were carried out on frog skin and the stripped gastric mucosa of the bull frog (Rana catesbeiana). These isolated membranes were mounted in a modified Ussing-type chamber apparatus (Fig. 2). The orifice of the chamber was $11.3 \mathrm{~mm}$ in diameter and about $1.0 \mathrm{~cm}^{2}$ in

Fig. 2

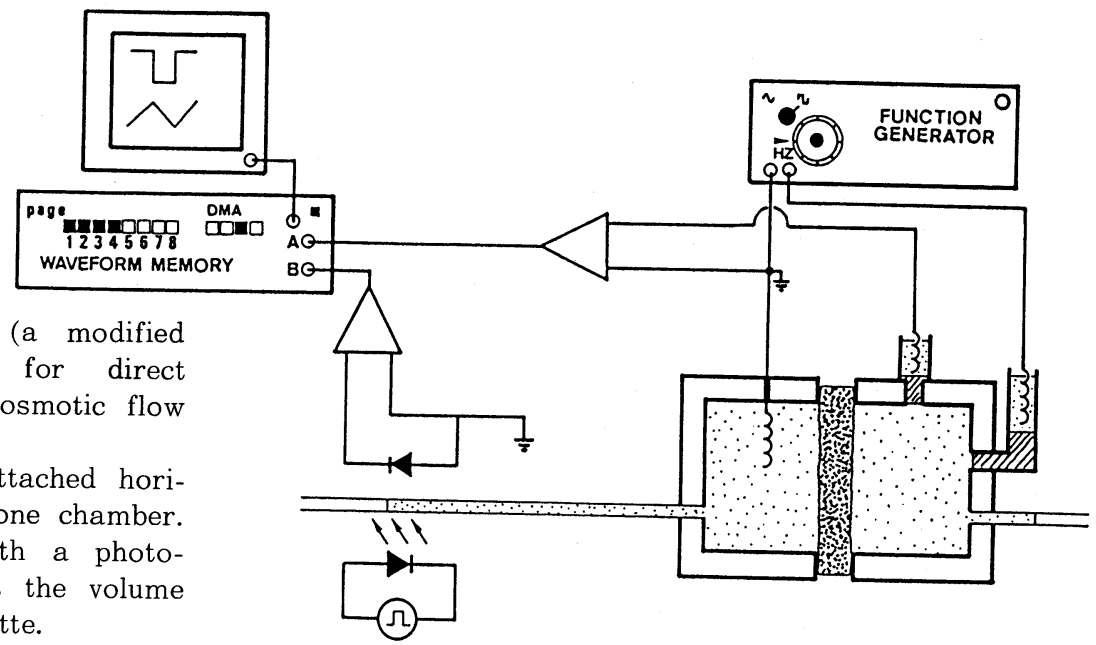

Experimental apparatus (a modified Ussing-type chamber) for direct measurement of electro-osmotic flow with a volumetric device.

A glass pipette is attached horizontally to the side of one chamber. A volumetric device with a photosensitive diode measures the volume changes in the glass pipette. 
area. The volume of each chamber was $4.5 \mathrm{ml}$. One side of the chamber was covered with nylon mesh to reinforce the mechanical movement of the mounted membrane ${ }^{3,4)}$. A glass pipette with inner diameter of $0.8 \mathrm{~mm}$ was attached horizontally to the closed chamber. A volumetric device with a photodiode was used to monitor volume flow in the glass pipette. This photosensitive volumetric device was set in a photoshielded acryl box with a narrow slit $2 \times 20 \mathrm{~mm}$. Through the slit, an infrared radiative diode (GL 540 SHARP) faced the photosensitive diode (SPD 540 SHARP). A photo-induced current was generated in proportion to the fluid volume in the pipette. This volumetric device can detect volume change from 0 to $4 \mu \mathrm{l}$ ( $8 \mathrm{~mm}$ long) with accuracy of $0.02 \mu \mathrm{l}(0.04 \mathrm{~mm}$ long). The other chamber was connected to two electrodes through agar bridges, one for measuring the difference in potential and the other for applying an electric current. The electric potential was monitored in this chamber with reference to an $\mathrm{Ag}-\mathrm{AgCl}$ electrode in the volumetric chamber. The solution used was $10 \mathrm{mM}$ HEPES frog Ringer solution ( $\mathrm{pH} 7.4)$, which contained $\mathrm{Na}^{+}$ $115 \mathrm{mEq} / 1, \mathrm{~K}^{+} 2.5 \mathrm{mEq} / 1, \mathrm{Ca}^{2+} 1.1 \mathrm{mEq} / 1, \mathrm{Cl}^{-} 115$ $\mathrm{mEq} / \mathrm{l}, \mathrm{H}_{2} \mathrm{PO}_{4}^{-} 0.08 \mathrm{mEq} / \mathrm{l}$, glucose $11.0 \mathrm{mM}$ and HEPES $10 \mathrm{mM}$. Amiloride $10^{-5} \mathrm{M}$ was used for active $\mathrm{Na}$ transport block for frog skin.

Electric potential was oscillated by a function generator (MODEL 459 KIKUSUI Co.) and measured with a digital multimeter (TAKEDA RIKEN TR-2112A). These data were recorded on a waveform memory (WAVEFORM MEMORY MODEL 8100 KIKUSUI).

\section{Results}

\section{Direct Measurements of electro-osmotic volume flow}

When the membrane potential across the frog skin was changed externally by applying rectangular potential waves, the volume flow oscillated synchronously. The synchronized volume a) skin

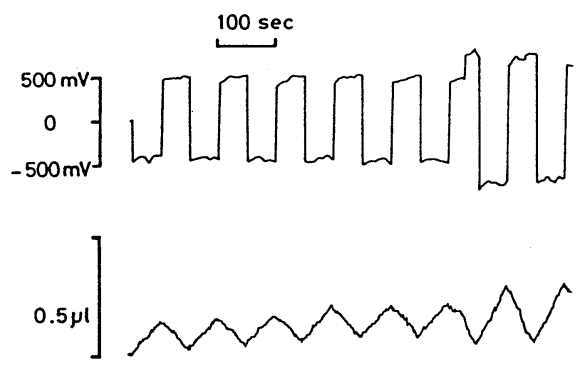

b) gastric mucosa

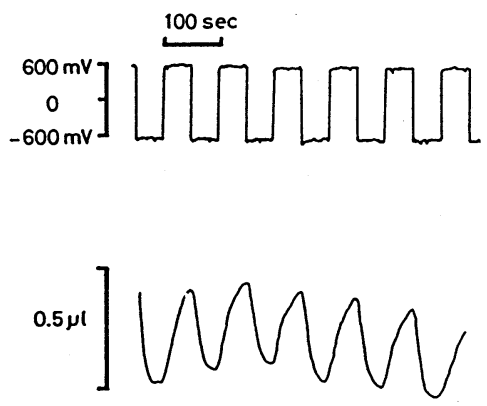

Fig. 3 Results of electro-osmotic flow measurements

In the frog skin (a), when the membrane potential was changed externally by applying alternating rectangular potentials, the volume flow oscillated synchronously (150nl/ $\min , \pm 500 \mathrm{mV})$. By the end of tracing, the applied potential was increased, and the flow response was also increased $(220 \mathrm{nl} / \mathrm{min}, \pm 750 \mathrm{mV})$. In the gastric mucosa (b), the volume flow response was larger $(400 \mathrm{nl} / \mathrm{min}$, $\pm 600 \mathrm{mV})$. In the frog skin, the volume flow was in the direction of cation flow. In the gastric mucosa, the volume flow was in the direction of anion flow.

flow oscillation in the frog skin caused by the rectangular waves was shown in Fig.3-a. During the positive potential $(500 \mathrm{mV})$, the volume flow rose into the pipette. The flow rate was $150 \mathrm{nl} /$ $\mathrm{min} / \mathrm{cm}^{2}$, and expressed as a downward slope. But during the negative potential $(-500 \mathrm{mV})$, the volume flow was in the opposite direction at a 
rate of $150 \mathrm{nl} / \mathrm{min} / \mathrm{cm}^{2}$, and expressed as an uphill slope. When the amplitude of the potential wave increased to $\pm 750 \mathrm{mV}$ at the end of the tracing, the volume flow increased to $220 \mathrm{nl} / \mathrm{min} /$ $\mathrm{cm}^{2}$. The direction of volume flow changed without time delay, when the electric potential was changed.

The gastric mucosa showed a larger response $\left(400 \mathrm{nl} / \mathrm{min} / \mathrm{cm}^{2}, \pm 600 \mathrm{mV}\right)$ than the frog skin as shown in Fig.3-b. The phase relation between the applied potential and the volume flow response was also examined. The phase shift observed in the frog skin was the same as that of a cation exchange membrane, in which the volume flow was directed to the negative potential side (Fig. 3-a), while the phase shift of the gastric mucosa was the same as that of an anion exchange membrane, in which the volume flow was directed to the positive potential side (Fig. 3-b).

\section{Relationship between volume flow and amplitude} of applied potential (Fig. 4)

The volume flow increased in proportion to the amplitude of the applied potential for each membrane (Fig. 4-a, b). In this experiment, the direction of the volume flow into the pipette was expressed as positive. The regression lines were $Y=0.00031 \times X-0.0028$ for the $\operatorname{frog} \operatorname{skin}(n=6)$, and $Y=-0.00069 \times X-0.0025$ for the gastric mucosa $(n=3)$. The ratios of the volume flow to the applied potentials were $0.31 \mu \mathrm{l} / \mathrm{min} / \mathrm{V} / \mathrm{cm}^{2}$ for the frog skin, and $0.69 \mu \mathrm{l} / \mathrm{min} / \mathrm{V} / \mathrm{cm}^{2}$ for the gastric mucosa. The ratio of the gastric mucosa is twice than that of the skin. Spontaneous water flow was too small to measure in our apparatus, because these phenomena were very similar with or without amiloride $10^{-5} \mathrm{M}$.

Electro-osmosis against constant osmotic flow (Figs. 5, and 6)

In order to compare electro-osmotic flow with osmotic flow, rectangular potential wave was applied during a finite osmotic gradient. In this condition the values of hydraulic conductivity a) skin

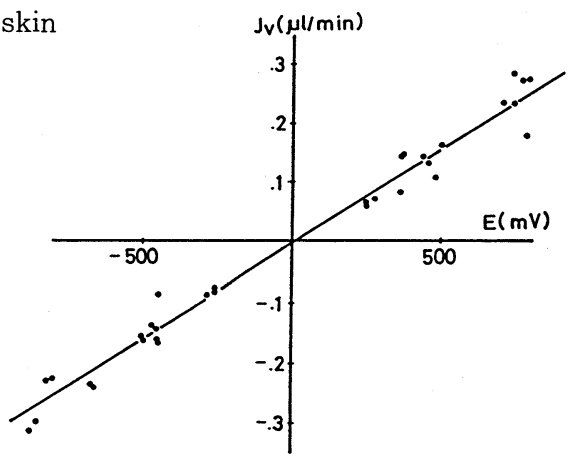

b) gastric mucosa

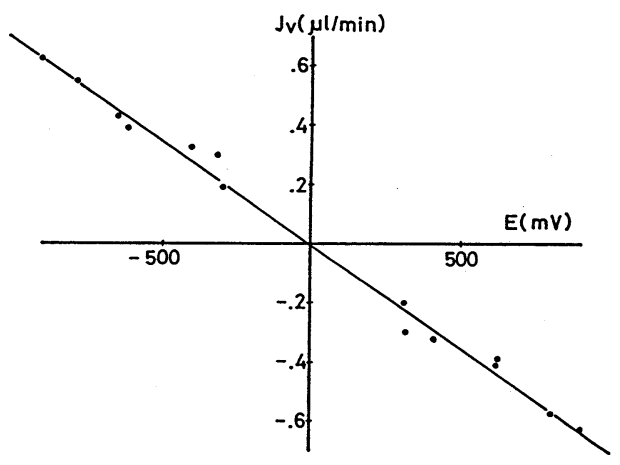

Fig. 4 Relationship between observed volume flow and amplitude of applied potential (Volume flow into the pipette is expressed as positive here.)

The induced volume flow was in proportion to the amplitude of the applied potential.

$(L p)$ and reflection coefficient $\left(\sigma_{i}\right)$ were estimated. The osmotic flow was driven by osmotic gradients of sucrose. Then the level of rectangular potential waves was increased stepwise as shown in Fig. 5-a, and the height of the potential needed to keep the volume flow zero was estimated. For the frog skin (Fig. 5-a), the osmotic gradient was made by adding $200 \mathrm{mOsm}$ of sucrose to HEPES-Ringer solution ( $\mathrm{pH} 7.4)$ on the volumetric side. At this stable osmotic flow, shown as a downward slope, rectangular waves of several potential levels across the membrane were applied. When the negative potential was increased, electro-osmotic flow was induced in the opposite direction and increased in proportion to the potential level. The net 
a)
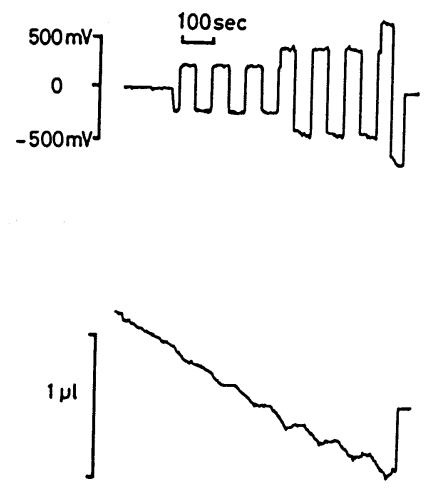

b)

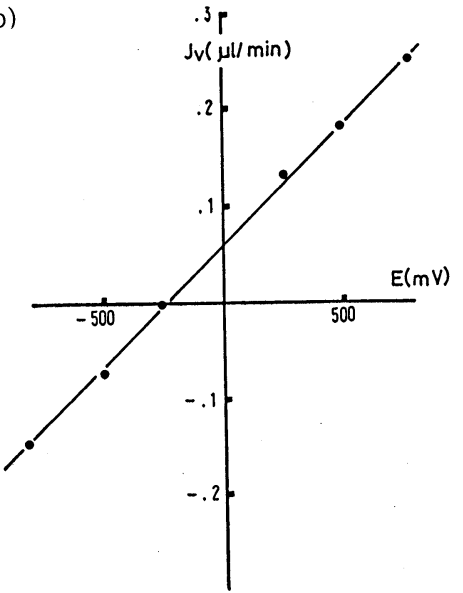

Fig.5 Electro-osmosis during constant osmotic gradient in frog skin.

a. The osmotic gradient was made by adding $200 \mathrm{mOsm}$ sucrose to $10 \mathrm{mM}$ HEPES Ringer solution ( $\mathrm{pH} 7.4)$ on the volumetric side. After ensuring constant osmotic flow (shown as a downhill slope), the transepithelial potential was oscillated by rectangular waves to evoke an electro-osmotic flow. The potential was increased stepwise to show how large a potential gradient was need to balance or reverse the finite osmotic flow. The induced electroosmotic flow decreased and even inverted the net volume flow.

b. Volume flow rate was calculated at each step of the electric potential. The relationship between the transepithelial potential and the volume flow was plotted. The regression line was $Y=0.00025 \times X+0.060$. The value of $X$ is the value of the potential which balances the finite osmotic flow.

volume flow decreased and finally turned upward as the applied potential increased. The relationship between volume flow rate and electric potential is shown in Fig. $5-\mathrm{b}$. When $J v$ was 0 , the value of $\Delta \varphi$ was $-241 \mathrm{mV}$, which was the electric potential balanced the $200 \mathrm{mOsm}$ osmotic flow. When $\Delta \varphi$ was 0 , the value of $J v$ was $0.06 \mu l / \mathrm{min} /$ $\mathrm{cm}^{2}$, which was the osmotic volume flow driven by $200 \mathrm{mOsm}$ sucrose. These relationships are expressed by the transport equation :

$$
J v=L p\left\{\left(-\Delta \pi_{\text {sucrose }}\right)+F \Delta \varphi \Sigma_{i}\left(1-\sigma_{i}\right) z_{i} c_{i}\right\}
$$

Here we assume that the electrolyte is single monovalent salt such as $\mathrm{NaCl}$; then $\bar{c}_{\text {anion }}=\bar{c}_{\text {cation }}$ $=c_{\text {salt }}$, and we obtain the following equation:

$$
\begin{aligned}
J v=L D\{( & \left(-\Delta \pi_{\text {sucrose }}\right)+ \\
& \left.c_{\text {salt }} F \Delta \varphi\left(\sigma_{\text {anion }}-\sigma_{\text {cation }}\right)\right\}
\end{aligned}
$$

When $J v=0$,

$$
\Delta \pi_{\text {sucrose }}=c_{\text {salt }} F \Delta \varphi\left(\sigma_{\text {anion }}-\sigma_{\text {cation }}\right)
$$

$\Delta \varphi=-241 \mathrm{mV}$ and $c_{\text {salt }}=0.115 \mathrm{mM}$, then the value of $\sigma_{\text {anion }}-\sigma_{\text {cation }}$ is 0.14 . $\sigma_{\text {anion }}$ is larger than $\sigma_{\text {cation }}$

When $\Delta \varphi=0$,

$$
J v=L p(-\Delta \pi \text { sucrose })
$$

Since $J v=0.06 \mu \mathrm{l} / \mathrm{min} / \mathrm{cm}^{2}$ in the case of $200 \mathrm{mOsm}$ sucrose gradient, $L p$ was estimated to be $2 \times 10^{-7}$ $\mathrm{cm} / \mathrm{sec} / \mathrm{atm}$.

For the gastric mucosa (Fig.6-a), the osmotic gradient was made by adding $100 \mathrm{mOsm}$ sucrose to HEPES-Ringer solution (pH7.4) on the volumetric side. On the stable osmotic flow, shown as a downward slope, rectangular waves of several potential levels were applied across the membrane. When level of the positive potential was increased, volume flow was turned to the opposite direction and became larger in proportion to the increment of potential level. The relationship between volume flow rate and electric potential is shown in Fig. 6-b. When Jv was 0 , the value of $\Delta \varphi$ was $156 \mathrm{mV}$, which was the electric potential balanced $100 \mathrm{mOsm}$ osmotic 
a)

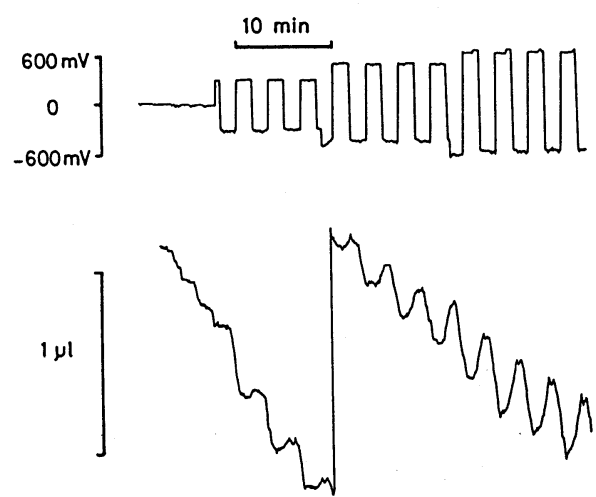

b)

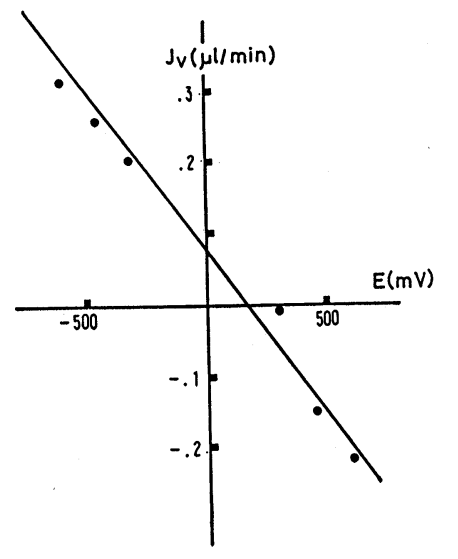

Fig. 6 Electro-osmosis during constant osmotic gradient in gastric mucosa.

a. The osmotic gradient was made by adding $100 \mathrm{mOsm}$ sucrose to $10 \mathrm{mM}$ HEPES Ringer solution $(\mathrm{pH} 7.4)$ on the volumetric side. Experimental procedure is as the same as shown in Fig. 5.

b. The relationship between the trasepithelial potential and the volume flow was plotted. The regression line was $Y=0.00042 \times X+0.066$.

flow. When $\Delta \varphi$ was 0 , the value of $J v$ was 0.066 $\mu \mathrm{l} / \mathrm{min} / \mathrm{cm}^{2}$, which was the osmotic volume flow driven by $100 \mathrm{mOsm}$ sucrose. These relationships are expressed by the transport equations (7), (8) and (9), as described above. When $J v=0, \Delta \varphi=$ $156 \mathrm{mV}$, then the value of $\sigma_{\text {anion }}-\sigma_{\text {cation }}$ is -0.11. $\sigma_{\text {cation }}$ is larger than $\sigma_{\text {anion. }}$.

When $\Delta \varphi=0, J v=0.066 \mu \mathrm{l} / \mathrm{min} / \mathrm{cm}^{2}$ in the case of 100 mOsm sucrose gradient, then the value of $L p$ is $4.5 \times 10^{-7} \mathrm{~cm} / \mathrm{sec} / \mathrm{atm}$.

\section{$\mathrm{Cl}^{-}$replaced by $\mathrm{SO}_{4}^{2-}$}

The experiment with $\mathrm{SO}_{4}^{2-}$ replacing $\mathrm{Cl}^{-}$was also performed with frog skin (Fig.7). $\mathrm{A} \mathrm{SO}_{4}$ Ringer solution was made by replacing $\mathrm{Cl}^{-}$ions with $\mathrm{SO}_{4}^{2-}$ ions in normal Ringer solution, and osmolarity was adjusted with sucrose. The control electro-osmotic flow was obtained by applying rectangular electric potential waves of $\pm 350 \mathrm{mV}$ in normal Ringer solution. In the $\mathrm{SO}_{4}$ Ringer solution, the volume flow response is larger than the case of $\mathrm{Cl}$-Ringer solution under the similar electric conditions. The ratio of the flow rates between in the case of Cl-Ringer solution and $\mathrm{SO}_{4}$-Ringer solution was $1.31 \pm 0.14$ $(n=6)$. a)
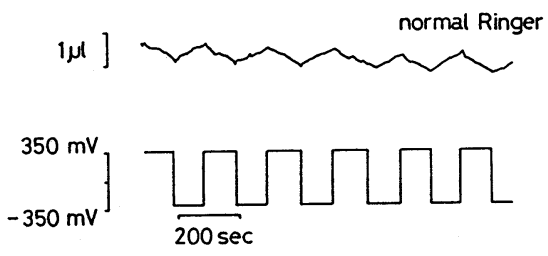

b)
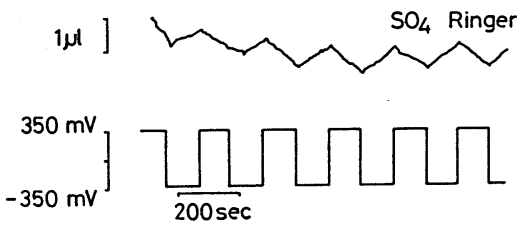

Fig.7 Effect of replacing $\mathrm{Cl}^{-}$with $\mathrm{SO}_{4}^{2-}$

The volume flow response (b) increased to $1.31 \pm 1.14$ times the control value (a) $(n=6)$.

In the sulfate Ringer solution,

$$
J v=L p\left\{\left(\sigma_{\mathrm{sO}_{4}}-\sigma_{\mathrm{Na}}\right) c F \Delta \varphi\right\}
$$

Here $c=\bar{c}_{\mathrm{Na}}=2 \bar{c}_{\mathrm{SO}_{4}}$. The above ratio of 1.31 is expressed as $\left(\sigma_{\mathrm{SO}_{4}}-\sigma_{\mathrm{Na}}\right) /\left(\sigma_{\mathrm{Cl}}-\sigma_{\mathrm{Na}}\right)$. If we assume $\sigma_{\mathrm{SO}_{4}}=1$, then $\sigma_{\mathrm{cl}}=0.96$ and $\sigma_{\mathrm{Na}}=0.82$.

\section{Discussion}

Electro-osmosis was considered to be small in biological membrane ${ }^{6 \sim 8)}$. But Hill assumed that electro-osmosis play an important role in theoretical model ${ }^{9,10)}$. We showed that electro- 
osmotic coupling is so important that it could not be ignored in evaluating water transport mechanism in epithelia ${ }^{1,2)}$. To analyse the electro-kinetic phenomena in epithelia, the oscillation method was used in á modified Ușsing chamber, where no chemical potential and no hydrostatic pressure exist.

In the theoretical model of network thermodynamics, Imai et $\mathrm{al}^{3,4)}$ described a bioenergy system which includes multi-power domains. They assumed that there are direct interactions between electric power and ionic power and between ionic power and hydrostatic power, but there are indirect interactions between electric power and hydrodynamic power and among various ionic powers. The electro-kinetic phenomenon, therefore, can be considered as a power coupling process between hydraulic and electric powers via ionic powers. Streaming potential is a power transducing process from hydraulic to electric power via ionic transport process. Electro-osmosis is a power transducing process from electric to hydraulic power via ionic transport process. When there are no differences in chemical potentials or osmotic pressure across the membrane, our simple model can be used to demonstrate - electro-kinetic phenomena including power transducing processes. As illustrated in Fig. 8, the membrane potential $\Delta \varphi$ reflects the ionic driving forces of cations and anions. Monovalent cations undergo a driving force of $F \Delta \varphi$ and monovalent anions undergo a force $-F \Delta \varphi$. The ionic driving forces interact with the hydraulic driving force as friction. This frictional force is $\left(1-\sigma_{\text {cation }}\right)$ $\bar{c} F \Delta \varphi$ in the case of cation, and $-\left(1-\sigma_{\text {anion }}\right)$ $\bar{c} F \Delta \varphi$ in the case of anion. The transmitted driving force of volume flow is $\left(\sigma_{\text {anion }}-\sigma_{\text {cation }}\right)$ $\bar{c} F \Delta \varphi$.

As expected from this thermodynamic approach, the induced volume flow increased in proportion to amplitude of the potential difference in our experiment. In $10 \mathrm{mM}$ HEPES Ringer

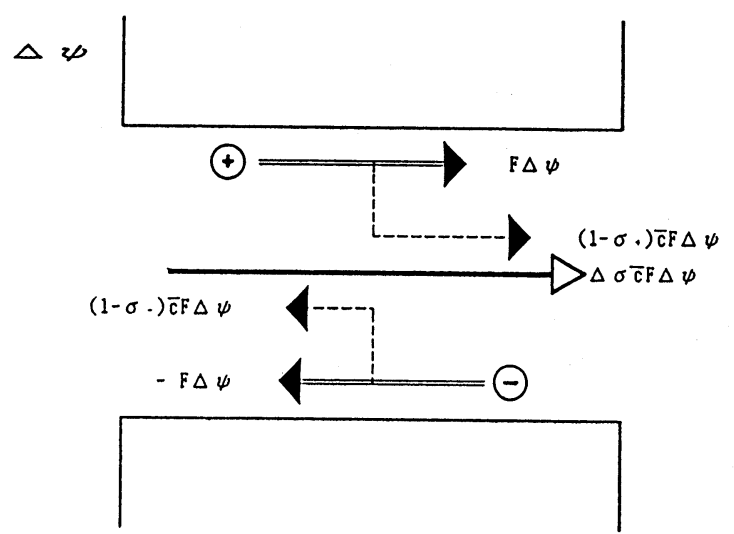

Fig. 8 Power Coupling

The potential difference $\Delta \varphi$ gives the driving force of $F \Delta \varphi$ to monovalent cations, and that of $-F \Delta \varphi$ to monovalent anions in the reverse direction. Each force is transmitted to the volume flow by friction.

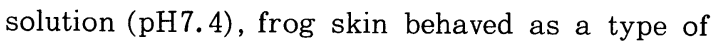
cation exchanger ${ }^{11,12)}$, and gastric mucosa behaved as a type of anion exchanger. The reflection coefficient reflected the charge and the size of the water channel, and the phase shift depended on the $\left(\sigma_{\text {anion }}-\sigma_{\text {cation }}\right)$. When $\sigma_{\text {anion }}$ is larger than $\sigma_{\text {cation, }}$ the water channel is negatively charged and behaves as a cation exchanger. The cationic driving force for the volume flow exceeded the anionic driving force for that in the frog skin, and the volume flow was concomitant with the cation flow. On the otherhand, when $\sigma_{\text {anion }}$ is smaller than $\sigma_{\text {cation, the }}$ water channel is positively charged and behaves as an anion exchanger. In the gastric mucosa, the anionic driving force for the volume flow exceeded the cationic driving force for that. The hydraulic driving force induced by ionic flows was concomitant with the anionic flow. The phase shift in the epithelia changed with $\mathrm{pH}$ of the solution, as reported by Mudd $(1925)^{13)}$, Wright \& Diamond $(1968)^{14)}$, Smyth \& Wright $(1966)^{15)}$ and Imai, et al $(1985)^{2}$.

Wedner and Diamond $(1969)^{6)}$ reported that a large component of the electro-kinetic phenome- 
non was owing to local concentration changes in the unstirred layer in their rabbit gallbladder experiments, and assumed that the concentration gradient formed in the unstirred layer was due to continuous volume flow and that the half time of its decay was $22-90 \mathrm{sec}^{12)}$. In practice, it is very difficult to eliminate a local concentration effect from electro-kinetic phenomena during constant changes of chemical potentials and osmotic pressure. But in our experiment, changes in volume flow direction occurred just after potential change. If there is some unstirred layer effects, a time delay would be reconized because of its long half time.

Combined experiments were performed with a constant osmotic flow to demonstrate true electro-osmosis. During the finite osmotic flow due to sucrose, the electro-osmotic flow induced by potential oscillation did noi only decrease but inverted the net volume flow. The response in gastric mucosa was twice that for frog skin, because the $L p$ of the former was larger than that of the latter. Our Lp values, $2 \times 10^{-7} \mathrm{~cm} / \mathrm{sec} /$ atm for skin and $4.5 \times 10^{-7} \mathrm{~cm} / \mathrm{sec} / \mathrm{atm}$ for gastric mucosa, are almost the same as $3.9 \times 10^{-7} \mathrm{~cm} / \mathrm{sec} /$ atm for the frog skm (R. temporalia) reported by House $(1964)^{161}$, and $8.1 \times 10^{-7} \mathrm{~cm} / \mathrm{sec} / \mathrm{atm}$ for frog gastric mucosa reported by Durbin, Frank and Solomon (1956) ${ }^{17}$ ) who used a conventional method of osmotic pressure measurement.

In this paper, the values of reflection coef- ficients were also estimated, that is, $\sigma_{\mathrm{cl}}=0.96$ and $\sigma_{\mathrm{Na}}=0.82$ when $\sigma_{\mathrm{sO}_{4}}=1$ for frog skin.

\section{Reference}

1) Y. Imai, M. Miyamoto, T. Nakahari M. Murakami and H. Yoshida: Jpn. J. Physiol., 36, 397 (1986)

2) Y. Imai, M. Miyamoto, T. Nakahari, H. Tanaka and Y. Soma: Biomedical Research 7, Supplement 2, 213 (1986)

3) Y. Imai, H. Yoshida and M. Murakami : Membrane, 10, 63 (1985)

4) Y. Imai, T. Nakahari, H. Mori and $H$. Yoshida: Membrane, 10, 127 (1985)

5) O. Kedem and A. Katchalsky: Biochim. Biophys. Acta, 27, 229 (1958)

6) H. J. Wedner and J. M. Diamond : J. Membrane Biol., 1, 92 (1969)

7) J. M. Diamond, W. H. Bossert : J. Gen . Physiol., 50, 2061 (1967)

8) J. M. Diamond: J. Physiol., 183, 58 (1966)

9) A. E. Hill : Proc. R. Soc., B 190, 99 (1975)

10) A. E. Hill : Proc. R. Soc., B 190, 115 (1975)

11) J. H. Moreno and J. M. Diamond : In "Membranes-A series of Advances" vol.3. Ed. G. Eisenman, Marcel Dekker, New York, 383 (1975)

12) J. M. Diamond and S. C. Harrison : J. Physiol., $183: 37$ (1966)

13) S. Mudd: J.Gen. Physiol., 7, 389 (1925)

14) E. M. Wright and J. M. Diamond: Biochem. Biophys. Acta , 163, 57 (1968)

15) D. H. Smyth and E. M. Wright: J. Physiol. (Lond.) 182, 591 (1966)

16) C. R. House: Biophysical Journal, 5, 987, 987 (1964)

17) R. P. Durbin, H. Frank and A. K. Solomon : J. of General Physiology, 39, 535 (1956) 